\section{THE ETIOLOGY OF CHOLERA.} By C. EGERTon FITZ-GERALD M.D. St. And.

THE discussion on the cause or causes of cholera and the great divergence of opinion existing thereon remind one forcibly of the celebrated discussion as to the colour of the chameleon, in which, as now, each observer saw and spoke from his individual experience. The question is, May they not all be right? Cholera, like influenza, will eventually be found to be a miasmatic disease, of which the hitherto undiscovered germ can be conveyed through the air, by water, excreta, infected bodies or clothing. What the special germ may be, we as yet know not; but that it multiplies with enormous rapidity under favourable conditions of heat, moisture and dirt there can be no doubt. Theoretically one would expect it to be some hardy microscopic spore, ever present in certain localities, and ready, under exceptional circumstances favourable to its growth and development, to be produced in enormous volumes, whether in impure air, polluted water, in sodden soil or infected bodies. We should thus expect it to follow occasionally the course of rivers, sometimes to descend in deadly clouds in one place, while it rose over others, as it was borne by varying air currents with apparent caprice. Each individual as he is attacked becomes a fresh nidus, a hotbed for disease germs, which seek and require only a suitable soil or cultivating medium for their propagation; but a suitable condition of the atmosphere exists only under certain exceptional circumstances. This accounts for the rapid spread of cholera amongst large masses, especially dirty masses of men. Each unit of infection acts on suitable media exactly as would a particle of yeast if introduced into a mass of fermentable fluid urder the requisite conditions of temperature \&c. This is the explanation of the fact that, although cholera may arise sporadically anywhere under favourable but exceptional circumstances, it is endemic only in India, where, presumably, those requisite conditions constantly prevail. That cholera does spread principally along the lines of human intercourse, that it may be conveyed by man, by water, by fomites, may be readily conceded without affecting the contention as to its miasmatic and aerial character and method of propagation. That cholera is caused by Koch's vibrio is to the last degree improbable and certainly unproven, although it may be admitted that the cholera bacillus is usually present in the dejecta ; but, setting aside the many morphologically similar varieties of this bacillus already described as being ever present in the intestines, what does this prove? Simply that in cholera, as in so many other diseases ascribed to microbes, we have the media, the special soil, suitable to its growth newly upturned earth the conditions favourable to the growth of the spores of the mushroom, or the seeds of the numerous weeds which so rapidly spring up and flourish. The universal germ theory, now so eagerly and enthusiastically accepted, is not more generally believed in than was the humoral theory or the dyscrasia of a former generation, yet who credits these now? And is it not possible that our successors may have to climb down from our present position in as rapid and undignified a manner as we have already done from the beliefs of the past? How, otherwise than by aerial and miasmatic infection, can we explain the abrupt termination of an outbreak of cholera on the supervention of frost, heavy rain, or a high wind? How account for the sudden simultaneous visitation, or the as sudden cessation, of cholera in two villages separated by many miles of intervening valley (as recorded by Surgeon - Colonel Hamilton), its sudden appearance in ships at sea, in places where the water-supply is beyond reproach and where its importation by human agency is to the last degree improbable? 'l'he miasmatic theory perfectly fits in with and explains Pettenkofer's " subsoil water" hypothesis, for it is just when the surface water has sunk below its ordinary level that the very conditions of moisture, heat and evaporation most suitable for the growth and propagation of miasmatic spores are present. Nor does this supposition clash with the fact that cholera is communicable by drinking-water when contaminated with cholera germs. Supposing that water to be of the right temperature and to contain fermentable matter, it will prove an excellent culovating medium for the development of cholera germs, but should the temperature of the water fall below a certain point it will soon become innocuous. To my mind, all evidence tends to show that cholera is caused by some miasmatic germ which can be disseminated in a variety of ways, that the contagium is generally airborne, and that the comma bacillus has no etiological connexion with it, but flourishes only in the excreta because it there finds a congenial soil.

Folkestone.

\section{ASCARIDES LUMBRICOIDES. BY HENRY ALSTON, M.B., C.M., TRINIDAD MEDICAL SERYICE.}

IT would appear from cases recorded in THE LANCET lately that two distinct opinions exist amongst medical men as to the injurious effects of ascarides in the intestines. Amongst some the belief prevails that worms may cause death by entering the air passages or other parts, and possibly by producing mechanical obstruction of the bowels or enteritis, but that death from reflex irritation does not occur. On the other hand, medical men are found who insist on the importance of the reflex irritation alleged to be set up in the bowels by the worms; and the West Indies are stated to be the region where cases of this irritation are most frequent.

Some practitioners assert that the worms constitute the primary cause, any debility or blood deterioration being the consequence; while others maintain that the presence of the worms is but an indication of the lowering of the vitality of the mucous membrane of the stomach and bowels, as occurs in mal-nutrition and certain blood diseases, and the ascarides are simply a complication. This latter view is supported by the germ theory of constitutional diseases, which assumes not only the presence of the germs but the preparation of the host to receive it. Although the possibility of worms passing into the air passages and cansing death is admitted, it is necessary to remember that worms crawl out of the stomach after the death of the host. It is also necessary to recall the fact that intussusception and volvulus may be ante-mortem phenomena.

It will be interesting to put aside the question of marked pathological changes found associated with worms and let the thesis be "death from reflex irritation of ascarides." By reflex irritation is meant, I presume, death not from a single cause but from collapse or from convulsions and coma. The difficulties surrounding a belief in reflex irritation are as follows :-1. The intestinal mucous membrane is liable to the irritation of scybala and all sorts of foreign bodies swallowed by children and others accidentally. 2 . When strong purgatives are given to children considerable congestion and irritation of the bowels are set up, and yet death from reflex action is not recorded. 3. Diarrhoea ought to be a marked symptom of worms, and yet constipation is often found. 4. Opinions differ as to the number of worms required to produce the irritation. Will six worms do it? or does it require forty or more? 5. In intussusception affecting children great congestion and irritation occur, yet reflex convulsions and coma are not recorded as characteristic signs. 6. In malarious districts there is a form of malarial ferer, called in the text-books "pernicious intermittent," that can cause death in a few hours. Convulsions in the case of children take the place of the cold stage, and the marked collapse, with cold extremities, makes the relatives beliere that the child has no fever. There are also other well-known causes of convulsions in children, as anæmia with cerebral effusion \&c. In order to establish clearly at a post-mortem examination that death was due to reflex irritation from worms, the following points must be kept in view :--(a) An examination of the brain is necessary ; $(b)$ cerebral congestion must be found with no indication of anæmia and enlarged spleen (for then the effusion is easily accounted for); (c) the history must exclude the possibility of a sharp attack of fever followed by collapse ; $(d)$ as cases occur when, on making a post-mortem examination, no clear indication of the cause of death is found (not even worms), an allow. ance must be made for such "unknown causes." Although I am not prepared to attribute to worms the rôle of producing death by reflex irritation, and I consider that it is rare for them to cause death in other ways, $I$ nevertheless believe that it is not only imperative to expel them from the bowels, but that to do it establishes confidence 